ISSN 1936-5098

CAE Working Paper \#08-08

\title{
Random Iterates of Monotone Maps
}

by

Rabi Bhattacharya
and
Mukul Majumdar

December 2008 


\section{Editorial Manager(tm) for Review of Economic Design \\ Manuscript Draft}

Manuscript Number: ROED-D-07-00023

Title: Random Iterates of Monotone Maps

Article Type: Special Issue for Dr. Hurwicz

Section/Category:

Keywords: Random Dynamical Systems, Invariant Distribution, Convergence, Markov Processes

Corresponding Author: Professor Mukul Majumdar,

Corresponding Author's Institution: Cornell University

First Author: Mukul Majumdar

Order of Authors: Mukul Majumdar; Rabi Bhattacharya

Manuscript Region of Origin:

Abstract: In this paper we prove the existence, uniqueness and stability of the invariant distribution of a random dynamical system in which the admissible family of laws of motion consists of monotone maps from a closed subset of a finite dimensional Euclidean space into itself. 


\title{
Random Iterates of Monotone Maps
}

\author{
Rabi Bhattacharya \\ Department of Mathematics \\ University of Arizona \\ Tucson, AZ 95721 \\ and \\ Mukul Majumdar \\ Department of Economics \\ Cornell University \\ Ithaca, NY 14853
}

June 7, 2007 


\section{Introduction}

This paper is dedicated to Leonid Hurwicz. Hurwicz (1944) was a contributor to the literature on stochastic models of growth and cycles. In collaboration with Kenneth Arrow he also set the tenor of research on multi-sector dynamic models [see Part III of Arrow and Hurwicz (1977)]. We focus on a class of stochastic or random dynamic processes that have been of particular interest in the context of optimization problems in - to use his terminology - "non-classical" environments. A formal statement of the main result is in Section 2. But we begin with a few informal remarks to provide the motivation. The mathematical model of discounted stochastic dynamic programming has become the basic tool in exploring optimal decision making under uncertainty both at the micro and macro levels. In "classical" models, by imposing appropriate (strict) convexity, continuity and monotonicity properties on the primitives (technological constraints involved in specifying the law of motion, return functions...), one is able to assert that the optimal policy function is monotone and continuous. Once, however, one attempts to step out of the "classical" environment (for example, to allow for a Knightian S-shaped production function that exhibits an initial phase of increasing returns), the standard proof of continuity of the optimal policy function fails. Indeed, even in a deterministic non-classical model of intertemporal optimization, an example of discontinuity (in which the production function is S-shaped, the return function is linear) was given in Majumdar and Mitra (1983). However, in a large class of stochastic models one can still prove that there is an optimal policy function that is monotonic (see Majumdar, Mitra and Nyarko (1989) for an elaboration of the finer points of selection and a comprehensive account 
of dynamic optimization under uncertainty with non-concave production functions). This monotonicity property turns out to be crucial in making significant progress in understanding the evolution of an optimal process, and in establishing some long run convergence properties. The analysis is simpler when the state space is an interval (in the real line). Exploring the implications of monotonicity (with possible discontinuity) when the state space is a closed subset of a finite dimensional Euclidean space is the point of departure of this paper. Consider a random dynamical system $(S, \Gamma, P)$ where $S$ is the state space (for example, a closed subset of $\mathbb{R}^{k}, \Gamma$ an appropriate family of maps on $S$ into itself and $P$ is a probability measure on (some $\sigma$-field of) $\Gamma$.

The evolution of the system can be described as follows: initially, the system is in some state $x$; an element $\alpha_{1}$ of $\Gamma$ is chosen randomly according to the probability measure $P$ and the system moves to the state $X_{1}=\alpha_{1}(x)$ in period one. Again, independently of $\alpha_{1}$, an element $\alpha_{2}$ of $\Gamma$ is chosen according to the probability measure $P$ and the state of the system in period two is obtained as $X_{2}=\alpha_{2}\left(\alpha_{1}(x)\right)$. In general, starting from some $x$ in $S$, one has

$$
X_{n+1}(x)=\alpha_{n+1}\left(X_{n}(x)\right)
$$

where the maps $\left(\alpha_{n}\right)$ are independent and identically distributed according to the measure $P$. The initial point $x$ can also be chosen (independently of $\left(\alpha_{n}\right)$ ) as a random variable $X_{0}$. The sequence $X_{n}$ of states obtained in this manner is a Markov process and has been of particular interest in economics (and other disciplines).

For describing "convergence to a long run steady state", perhaps the most widely 
used results identify conditions under which there is some time invariant probability measure $\pi$ such that, no matter what the initial $x_{0}$ is, $X_{n}$ converges in distribution to $\pi$. In this case we say that the (Markov) process is stable in distribution.

\section{The Main Result}

In this section we extend an important old result of Dubins and Freedman (1966) on i.i.d. iterations of monotone maps to multi-dimensional state spaces, and improve upon some recent results in Bhattacharya and Majumdar [(1999), (2007)], by dispensing with the requirement of continuity of the maps. The state spaces of the Markov process we consider is assumed to be a subset of $\mathbb{R}^{k}(k \geq 1)$ satisfying the following assumption:

(A.1) $\quad S$ is either a closed subset of $\mathbb{R}^{k}$, or a Borel subset which can be made homeomorphic to a closed subset of $\mathbb{R}^{k}$, by means of a strictly increasing continuous map on $S$ into $\mathbb{R}^{k}$.

It may be noted that every rectangle $X_{j=1}^{k} I_{j}$, where $I_{j}$ 's are arbitrary nondegenerate sub-intervals of the real line $\mathbb{R}$ satisfies the assumption (A1). For, an interval $(a, b)(-\infty \leq a<b \leq \infty)$ is homeomorphic to $(-\infty, \infty)$ by an appropriate strictly increasing continuous map. An interval $(a, b](-\infty \leq a<b<\infty)$ is similarly homeomorphic to $(-\infty, 0]$, etc.

To define the Markov process, let $\Gamma$ be a set of measurable monotone maps $\gamma=$ $\left(\gamma_{1}, \gamma_{2}, \ldots, \gamma_{k}\right)$ on $S$ into $S$, under the partial order: $\mathbf{x} \leq \mathbf{y}$ if $x_{j} \leq y_{j}$ for $1 \leq j \leq k ; \mathbf{x}$ $=\left(x_{1}, \ldots, x_{k}\right), \mathbf{y}=\left(y_{1}, y_{2}, \ldots, y_{k}\right) \in \mathbb{R}^{k}$ (or $\left.S\right)$. That is, either $\gamma$ is monotone increasing: 
$\gamma(\mathbf{x}) \leq \gamma(\mathbf{y})$ if $\mathbf{x} \leq \mathbf{y}$, or $\gamma$ is monotone decreasing: $\gamma(\mathbf{y}) \leq \gamma(\mathbf{x})$ if $\mathbf{x} \leq \mathbf{y} ; \mathbf{x}, \mathbf{y}$ $\epsilon S$. Let $\Gamma$ be endowed with a $\sigma$-field $\mathcal{C}$, and let $Q$ be a probability measure on $(\Gamma, \mathcal{C})$. Consider a sequence of i.i.d. maps $\left\{\alpha_{n}: n \geq 1\right\}$ with common distribution $Q$, defined on a probability space $(\Omega, \Im, P)$. For purposes of measurability, assume that the map $(\gamma, \mathbf{x}) \rightarrow \gamma(\mathbf{x})$ on $\Gamma X S$ into $S$ is measurable with respect to the product $\sigma$-field $\mathcal{C} \otimes \mathcal{B}(S)$ on $\Gamma X S$ and the Borel $\sigma$-field $\mathcal{B}(S)$ on $S$. For each y $\epsilon S$, define the Markov process $\left\{X_{n}: n \geq 0\right\}$ by

$$
X_{0}=\mathbf{y}, \quad X_{1}=\alpha_{1} X_{0}, \ldots, X_{n}=\alpha_{n} X_{n-1}=\alpha_{n} \alpha_{n-1} \ldots \alpha_{1} X_{0}
$$

where $\alpha_{n} \alpha_{n-1} \ldots \alpha_{1}$ denotes composition of maps in the indicated order. In general, $X_{0}$ can be any random variable with values in $S$, independent of the sequence $\left\{\alpha_{n}\right.$ : $n \geq 1\}$. The transition probability of the Markov process is $p(\mathbf{x}, B)=P\left(\alpha_{1} \mathbf{x} \epsilon\right.$ $B)=Q(\{\gamma \epsilon \Gamma: \gamma \mathbf{x} \in B\})$. In general, the $n$-step transition probability is given by the distribution of $X_{n}(\mathbf{x}) \equiv \alpha_{n} \alpha_{n-1} \ldots \alpha_{1} \mathbf{x}$, and is denoted by $p^{(n)}(\mathbf{x},$.$) . It may also$ be expressed as

$$
p^{(n)}(\mathbf{x}, B)=Q^{n}\left(\left\{\boldsymbol{\gamma} \in \Gamma^{n}: \tilde{\gamma} \mathbf{x} \in B\right\}\right), \quad(\mathbf{x} \in S, B \in \mathcal{B}(S)), \quad n \geq 1
$$

where $Q^{n}$ is the product probability on the product space $\left(\Gamma^{n}, \mathcal{C}^{\otimes n}\right)$, and $\tilde{\gamma}$ is the composition

$$
\tilde{\gamma} \mathbf{x}=\gamma_{n} \gamma_{n-1} \ldots \gamma_{1} \mathbf{x}\left(\gamma=\left(\gamma_{1}, \gamma_{2}, \ldots, \gamma_{n}\right) \epsilon \Gamma^{n}\right)
$$

Recall that $\pi$ is an invariant probability for the Markov process, or for the transition 
probability $p$, if $\pi$ is a probability measure on $(S, \mathcal{B}(S))$ satisfying

$$
\pi(B)=\int p(\mathbf{x}, B) \pi(d \mathbf{x}) \quad \forall B \in \mathcal{B}(S)
$$

In turn, (2.4) implies that $\pi(B)=\int p^{(n)}(\mathbf{x}, B) \pi(d \mathbf{x}) \forall B \in \mathcal{B}(S)$, and $\forall n \geq 1$. If one denotes the distribution of $X_{n}$ as $T^{* n} \mu$, where $\mu$ is the distribution of $X_{0}$, then $T^{* n}$ is the $n$-fold composition of $T^{*}: T^{* n}=T^{*} T^{*(n-1)}(n \geq 2), T^{* 1}=T^{*}$. Note that $T^{*}$ (as well as $T^{* n}$ ) is a map on the space $\wp(S)$ of all probability measures on $(S, \mathcal{B}(S))$ :

$$
\left(T^{* n} \mu\right)(B)=\int p^{(n)}(\mathbf{x}, B) \mu(d \mathbf{x}) \quad(\mu \epsilon \wp(S), B \in \mathcal{B}(S)) .
$$

Clearly, an invariant probability $\pi$ is just a fixed point of $T^{*} \equiv T^{* 1}$, in which case it is a fixed point of $T^{* n}$ for every $n$.

On the space $\wp(S)$, define, for each $a>0$, the metric

$$
d_{a}(\mu, \nu)=\sup _{g \in \mathcal{G}_{a}}\left|\int g d \mu-\int g d \nu\right|,(\mu, \nu \in \wp(S))
$$

where $\mathcal{G}_{a}$ is the class of all Borel measurable monotone (increasing or decreasing) functions $g$ on $S$ into $[0, a]$. It is simple to check that (i) $d_{a}=a d_{1}$, and (ii) the distance (2.6) remains the same if $\mathcal{G}_{a}$ is restricted to monotone increasing Borel measurable functions on $S$ into $[0, a]$. The following result is due to Chakraborty and Rao (1998), who derived a number of interesting results on the metric space $\left(\wp(S), d_{a}\right)$. One can show that convergence in the metric $d_{a}$ implies weak convergence if (A1) holds (see Bhattacharya and Majumdar (2007), pp. 287-288). 
Lemma 1 Under the hypothesis (A.1), $\left(\wp(S), d_{a}\right)$ is a complete metric space.

The following splitting condition generalizes that in Dubins and Freedman (1966). To state it, let $\tilde{\gamma}$ be as in (2.3), but with $n=N: \tilde{\gamma}=\gamma_{N} \gamma_{N-1} \ldots \gamma_{1}$ for $\gamma=$ $\left(\gamma_{1}, \gamma_{2}, \ldots, \gamma_{N}\right) \in \Gamma^{N}$

(A.2) There exist $F_{i} \in \mathcal{C}^{\otimes N}(i=1,2)$ for some $N \geq 1$, such that

(i) $\quad \delta_{i} \equiv Q^{N}\left(F_{i}\right)>0(i=1,2)$, and

(ii) for some $\mathbf{x}_{\mathbf{0}} \epsilon S$, one has

$$
\begin{aligned}
& \tilde{\gamma}(\mathbf{x}) \leq \mathbf{x}_{0} \quad \forall \mathbf{x} \epsilon S, \quad \forall \boldsymbol{\gamma} \epsilon F_{1}, \\
& \tilde{\gamma}(\mathbf{x}) \geq \mathbf{x}_{0} \quad \forall \mathbf{x} \in S, \quad \forall \boldsymbol{\gamma} \in F_{2},
\end{aligned}
$$

Also, assume that the set $H_{+}=\left\{\gamma \in \Gamma^{N}: \tilde{\gamma}\right.$ is monotone increasing $\} \in \mathcal{C}^{\otimes N}$.

Readers interested in the verification of the splitting condition in dynamic models in economics may turn to Bhattacharya and Majumdar (2007).

Our main result is the following:

Theorem 2 Let $\left\{\alpha_{n}: n \geq 1\right\}$ be a sequence of i.i.d. measurable monotone maps with a common distribution Q. Assume (A.1), (A.2) hold. Then there exists a unique invariant probability for the Markov process (2.1) and

$$
\sup _{\mathbf{x} \in S} d_{1}\left(p^{(n)}(\mathbf{x}, .), \pi\right) \leq(1-\delta)^{\left[\frac{n}{N}\right]}(n \geq 1)
$$

where $\delta=\min \left\{\delta_{1}, \delta_{2}\right\}$, and $\left[\frac{n}{N}\right]$ is the integer part of $\frac{n}{N}$. 
Proof. The proof uses Lemma (2.1) and two steps. The first involves detailed calculations.

Step 1. $T^{* N}$ is a uniformly strict contraction on $\left(\wp(S), d_{1}\right)$

Let $F_{i+}=F_{i} \cap H_{+}, F_{i-}=F_{i} \cap H_{-}$, where $H_{+}$is defined in (A.2), and $H_{-}=$ $\Gamma^{N} \backslash H_{+}(i=1,2)$. Define, for any given $g \in \mathcal{G}_{1}$, the functions

$$
\begin{aligned}
h_{i+}(\mathbf{x}) & =\int_{F_{i+\backslash} \backslash\left(F_{i+} \cap F_{j}\right)} g(\tilde{\boldsymbol{\gamma}} \mathbf{x}) Q^{N}(d \boldsymbol{\gamma}), \\
h_{i-}(\mathbf{x}) & =\int_{F_{i-\backslash\left(F_{i-} \cap F_{j}\right)} g(\tilde{\boldsymbol{\gamma}} \mathbf{x}) Q^{N}(d \boldsymbol{\gamma}), \quad(i=1,2 ; j \neq i) ;} g(\tilde{\boldsymbol{\gamma}} \mathbf{x}) Q^{N}(d \boldsymbol{\gamma}), \\
h_{3+}(\mathbf{x}) & =\int_{H_{+} \cap\left(F_{1} \cup F_{2}\right)^{c}} g(\tilde{\boldsymbol{\gamma}} \mathbf{x}) Q^{N}(d \boldsymbol{\gamma}), \\
h_{3-}(\mathbf{x}) & =\int_{H-\cap\left(F_{1} \cup F_{2}\right)^{c}} g(\tilde{\boldsymbol{\gamma}} \mathbf{x}) Q^{N}(d \boldsymbol{\gamma}) . \\
h_{4}(\mathbf{x}) & =\int_{F_{1} \cap F_{2}}
\end{aligned}
$$

Then the functions $h_{i \pm}(i=1,2,3)$, are monotone. To see this, let $g$ be monotone increasing, then $h_{i+}(i=1,2,3)$ are monotone increasing while $h_{i-}(i=1,2,3)$ are monotone decreasing. If $g$ is monotone decreasing, then the reverse holds. Now, 
for $g$ monotone increasing $\left(g \in \mathcal{G}_{1}\right)$,

$$
\begin{aligned}
& h_{1+}(\mathbf{x}) \leq g\left(\mathbf{x}_{0}\right)\left(Q^{N}\left(F_{1+}\right)-Q^{N}\left(F_{1+} \cap F_{2}\right)\right) \equiv a_{1+}, \\
& h_{1-}(\mathbf{x}) \leq g\left(\mathbf{x}_{0}\right)\left(Q^{N}\left(F_{1-}\right)-Q^{N}\left(F_{1-} \cap F_{2}\right)\right) \equiv a_{1-}, \\
& h_{2+}(\mathbf{x}) \geq g\left(\mathbf{x}_{0}\right)\left(Q^{N}\left(F_{2+}\right)-Q^{N}\left(F_{2+} \cap F_{1}\right)\right), \\
& h_{2-}(\mathbf{x}) \geq g\left(\mathbf{x}_{0}\right)\left(Q^{N}\left(F_{2-}\right)-Q^{N}\left(F_{2-} \cap F_{1}\right)\right), \\
& h_{3+}(\mathbf{x}) \leq Q^{N}\left(H_{+} \cap\left(F_{1} \cup F_{2}\right)^{c}\right) \equiv a_{3+}, \\
& h_{3-}(\mathbf{x}) \leq Q^{N}\left(H_{-} \cap\left(F_{1} \cup F_{2}\right)^{c}\right) \equiv a_{3-} .
\end{aligned}
$$

Also, write

$$
\begin{aligned}
& h_{2+}^{\prime}(\mathbf{x})=\int_{F_{2+} \backslash\left(F_{2+} \cap F_{1}\right)}(1-g(\tilde{\boldsymbol{\gamma}} \mathbf{x})) Q^{N}(d \boldsymbol{\gamma}), \\
& h_{2-}^{\prime}(\mathbf{x})=\int_{F_{2-} \backslash\left(F_{2-} \cap F_{1}\right)}(1-g(\tilde{\boldsymbol{\gamma}} \mathbf{x})) Q^{N}(d \boldsymbol{\gamma}) .
\end{aligned}
$$

Then $h_{2 \pm}^{\prime}(\mathbf{x})$ are monotone and satisfy

$$
\begin{aligned}
& h_{2+}^{\prime}(\mathbf{x}) \leq\left(1-g\left(\mathbf{x}_{0}\right)\right)\left(Q^{N}\left(F_{2+}\right)-Q^{N}\left(F_{2+} \cap F_{1}\right)\right) \equiv a_{2+}, \\
& h_{2-}^{\prime}(\mathbf{x}) \leq\left(1-g\left(\mathbf{x}_{0}\right)\left(Q^{N}\left(F_{2-}\right)-Q^{N}\left(F_{2-} \cap F_{1}\right)\right) \equiv a_{2-} .\right.
\end{aligned}
$$

Thus $h_{1 \pm} \epsilon \mathcal{G}_{a_{1} \pm}, h_{2 \pm}^{\prime} \epsilon \mathcal{G}_{a_{2 \pm}}, h_{3 \pm} \epsilon \mathcal{G}_{a_{3 \pm}}$. Also,

$$
\left|\int h_{2 \pm}(\mathbf{x}) \mu(d \mathbf{x})-\int h_{2 \pm}(\mathbf{x}) \nu(d \mathbf{x})\right|=\left|\int h_{2 \pm}^{\prime}(\mathbf{x}) \mu(d \mathbf{x})-\int h_{2 \pm}^{\prime}(\mathbf{x}) \nu(d \mathbf{x})\right|,
$$


and

$$
\int h_{4}(\mathbf{x}) \mu(d \mathbf{x})-\int h_{4}(\mathbf{x}) \nu(d \mathbf{x})=0, \quad(\mu, \nu \epsilon \wp(S)) .
$$

The last relation follows from the fact that $h_{4}(\mathbf{x})=g\left(\mathbf{x}_{0}\right) Q^{N}\left(F_{1} \cap F_{2}\right)$, a constant function on $S$. For, on $F_{1} \cap F_{2}, \tilde{\gamma}(\mathbf{x})=\mathbf{x}_{0} \forall \mathbf{x} \epsilon S$. Hence

$$
\begin{aligned}
& \left|\int g d\left(T^{* N} \mu\right)-\int g d\left(T^{* N} \nu\right)\right| \\
= & \mid \sum_{i=1}^{3}\left[\int h_{i+}(\mathbf{x}) \mu(d \mathbf{x})-\int_{i} h_{i+}(\mathbf{x}) \nu(d \mathbf{x})+\int h_{i-}(\mathbf{x}) \mu(d \mathbf{x})-\int h_{i-}(\mathbf{x}) \nu(d(\mathbf{x})] \mid(2.14)\right. \\
\leq & \sum_{i=1,3}\left|\int h_{i+}(\mathbf{x}) \mu(d \mathbf{x})-\int h_{i+}(\mathbf{x}) \nu(d \mathbf{x})\right|+\sum_{i=1,3}\left|\int h_{i-}(\mathbf{x}) \mu(d \mathbf{x})-\int h_{i-}(\mathbf{x}) \nu(d \mathbf{x})\right| \\
& +\left|\int h_{2+}^{\prime}(\mathbf{x}) \mu(d \mathbf{x})-\int h_{2+}^{\prime}(\mathbf{x}) \nu(d \mathbf{x})\right|+\left|\int h_{2-}^{\prime}(\mathbf{x}) \mu(d \mathbf{x})-\int h_{2-}^{\prime}(\mathbf{x}) \nu(d \mathbf{x})\right| \\
\leq & \left(a_{1+}+a_{1-}+a_{2+}+a_{2-}+a_{3+}+a_{3-}\right) d_{1}(\mu, \nu) \equiv \bar{b} d_{1}(\mu, \nu), \text { say. }
\end{aligned}
$$

Note that

$$
\begin{aligned}
a_{1}+a_{1-} & =g\left(\mathbf{x}_{0}\right)\left(Q^{N}\left(F_{1}\right)-Q^{N}\left(F_{1} \cap F_{2}\right)\right), \\
a_{2}+a_{2-} & =\left(1-g\left(\mathbf{x}_{0}\right)\right)\left(Q^{N}\left(F_{2}\right)-Q^{N}\left(F_{1} \cap F_{2}\right)\right), \\
a_{3+}+a_{3-} & =Q^{N}\left(\left(F_{1} \cup F_{2}\right)^{c}\right)=1-Q^{N}\left(F_{1}\right)-Q^{N}\left(F_{2}\right)+Q^{N}\left(F_{1} \cap F_{2}\right),
\end{aligned}
$$

so that, adding these terms, one gets

$$
\begin{aligned}
\bar{b} & =1-\left[\left(1-g\left(\mathbf{x}_{0}\right)\right) Q^{N}\left(F_{1}\right)+g\left(\mathbf{x}_{0}\right) Q^{N}\left(F_{2}\right)\right] \\
& \leq 1-\min \left\{Q^{N}\left(F_{1}\right), Q^{N}\left(F_{2}\right)\right\}=1-\delta .
\end{aligned}
$$


Taking the supremum over all monotone increasing $g \in \mathcal{G}_{1}$ on the left in $(2.14)$, one arrives at the inequality

$$
d_{1}\left(T^{* N} \mu, T^{* N} \nu\right) \leq(1-\delta) d_{1}(\mu, \nu), \forall \mu, \nu \in \wp(S)
$$

Note that, the supremum in (2.6) over all of $\mathcal{G}_{a}$ is the same as the supremum over the subset of all monotone increasing functions in $\mathcal{G}_{a}$, since $a-g \epsilon \mathcal{G}_{a}$ and is monotone increasing if $g$ is monotone decreasing, $g \in \mathcal{G}_{a}$. Thus $T^{* N}$ is a uniformly strict contraction on $\left(\wp(S), d_{1}\right)$.

Step 2. Application of the Contraction Mapping Theorem.

From (2.16) and Lemma 2.1, it follows by the contraction mapping theorem that $T^{* N}$ has a unique fixed point $\pi$ in $\wp(S)$ and that, writing $n=\left[\frac{n}{N}\right] N+r$, one has

$$
\begin{aligned}
d_{1}\left(T^{* n} \mu, \pi\right) & =d_{1}\left(T^{*\left[\frac{n}{N}\right] N} T^{* r} \mu, T^{*\left[\frac{n}{N}\right] N} \pi\right) \\
& \leq(1-\delta)^{\left[\frac{n}{N}\right]} d_{1}\left(T^{* r} \mu, \pi\right) \leq(1-\delta)^{\left[\frac{n}{N}\right]} \forall \mu, \nu \in \wp(S) .
\end{aligned}
$$

In particular, (2.7) follows by letting $\mu=\delta_{\{\mathbf{x}\}}-$ the Dirac measure at $\mathbf{x}$ in $(2.17)$. Note that $T^{* N}\left(T^{*} \pi\right)=T^{*}\left(T^{* N} \pi\right)=T^{*} \pi$, so that $T^{*} \pi$ is also a fixed point of $T^{* N}$. By uniqueness of the fixed point $T^{*} \pi=\pi$.

Remark 2.1. In order to derive confidence regions of (or tests for) useful functionals of $\pi$ (e.g., the mean or dispersion), based on a finite set of observations $X_{j}(1 \leq j \leq n)$, one needs to derive asymptotic distributions of the corresponding functionals of the empirical distribution $\frac{1}{n} \sum_{j=1}^{n} \delta_{X_{j}}$. As in Bhattacharya and Majumdar [(2007), Sections 5.3, 5.4], one can show that, under the assumptions (A.1), 
(A.2), for every bounded function $g$ on $S$ which may be expressed as the difference $g_{1}-g_{2}$ of two bounded measurable monotone functions (or, equivalenty, for every finite linear combination of monotone functions), the central limit theorem (CLT) holds for its empirical mean $\frac{1}{n} \sum_{j=1}^{n} g\left(X_{j}\right)$, whatever the initial state. That is,

$$
\sqrt{n}\left(\frac{1}{n} \sum_{j=1}^{n} g\left(X_{j}\right)-\int g d \pi\right) \underset{n \rightarrow \infty}{\stackrel{\mathcal{L}}{\rightarrow}} N\left(0, \sigma^{2}\right)
$$

where $\stackrel{\mathcal{L}}{\rightarrow}$ denotes convergence in law, or distribution, and $N\left(0, \sigma^{2}\right)$ is the Normal distribution with mean 0 and variance $\sigma^{2}$. The variance parameter may be expressed as

$$
\sigma^{2}=\int f^{2}(\mathbf{y}) \pi(d \mathbf{y})-\int(T f)^{2}(\mathbf{y}) \pi(d \mathbf{y})
$$

where $T$ is the transition operator: $T h(\mathbf{x})=\int h(\mathbf{y}) p(\mathbf{x}, d \mathbf{y})$ and $f$ solves the Poisson equation in $L^{2}(S, \pi)$

$$
(I-T) f=g-\int g d \pi
$$

Here $L^{2}(S, \pi)$ is the Hilbert space of functions on $S$ which are square integrable (with respect to $\pi$ ). See Bhattacharya and Majumdar [(2007), Chapter 5] for more details on this general theme. In the case $S$ is non-compact and $g$ is unbounded (e.g., $g(\mathbf{x})=x_{j}$ for $\left.\mathbf{x}=\left(x_{1}, \ldots, x_{k}\right)\right)$, one requires that there exist a solution $f$ to the Poisson equation (2.20). Certain broad conditions for this solvability may be found in Bhattacharya and Lee (1988), for the case of i.i.d. monotone increasing maps.

Remark 2.2. Instead of the metric $d_{1}$, one may use a somewhat weaker metric 
$d_{\mathcal{A}}$ defined by

$$
d_{\mathcal{A}}(\mu, \nu)=\sup _{A \in \mathcal{A}}|\mu(A)-\nu(A)| \quad(\mu, \nu \in \wp(S)),
$$

where $\mathcal{A}$ comprises all sets of the form

$$
A=\{\mathbf{y} \epsilon S: \varphi(\mathbf{y}) \leq \mathbf{x}\}, \quad \mathbf{x} \epsilon S, \quad \varphi \text { monotone measurable. }
$$

One may prove the completeness of $\left(\wp(S), d_{\mathcal{A}}\right)$ more or less following the steps of the proof of Lemma C5.1, p. 287, in Bhattacharya and Majumdar (2007), where $\mathcal{A}$ is restricted to the class of sets $A$ in (2.22) with $\varphi$ continuous and monotone increasing. The analog of Theorem 2.2, with $d_{\mathcal{A}}$ in place of $d_{1}$, may then be proved roughly along the lines of the proof of Corollary 5.1, pp. 257-258, in Bhattacharya and Majumdar (2007). 


\section{References}

[1] Arrow, K.J. and Hurwicz, L. (1977): Studies in Resource Allocation Processes, Cambridge University Press, Cambridge.

[2] Bhattacharya, R.N. and Lee, O. (1988): "Asymptotics of a Class of Markov Processes which Are Not in General Irreducible", Annals of Probability, 16, 133347 (correction (1997): Annals of Probability, 25, 1541-43).

[3] Bhattacharya, R. and Majumdar, M. (2007) Random Dynamical Systems: Theory and Applications, Cambridge University Press, Cambridge.

[4] Bhattacharya, R. and Majumdar, M. (1999): "On a Theorem of Dubins and Freedman", Journal of Theoretical Probability, 12, 1067-1087.

[5] Chakraborty, S. and Rao, B.V. (1998): "Completeness of Bhattacharya Metric on the Space of Probabilities", Statistics and Probability Letters, 36, 321-326.

[6] Dubins, L.E. and Freedman, D.A. (1966): "Invariant Probabilities for Certain Markov Processes", Annals of Mathematical Statistics, 37, 837-868.

[7] Hurwicz, L. (1944): "Stochastic Models of Economic Fluctuations", Econometrica $12,114-124$.

[8] Majumdar, M. and Mitra, T. (1983): "Dynamic Optimization with Non-Convex Technology: The Case of a Linear Objective Function", Review of Economic Studies, 50, 143-151. 
[9] Majumdar, M., Mitra, T. and Nyarko, Y. (1989): "Dynamic Optimization Under Uncertainty: Non-Convex Feasible Set", Joan Robinson and Modern Economic Theory, (ed. G.R. Feiwel), MacMillan, 545-590. 